\title{
CABLE BASED ROBOTIC WORK PLATFORM FOR CONSTRUCTION
}

\author{
Thomas Richter' ${ }^{1}$, Steven J. Lorenc ${ }^{2}$, Leonhard E. Bernold ${ }^{3}$ \\ Construction Automation \& Robotics Laboratory \\ Department of Civil Engineering \\ North Carolina State University \\ Raleigh, NC 27695-7908 \\ sjlorenc@eos.ncsu.edu
}

\begin{abstract}
The following paper introduces a mechanism working as a suspension and positioning tool on the construction site. It is a highly variable tool which can be hooked to a wide range of cranes, where there is a stiff base from which to suspend a multitude of workcells. The design and control concepts are discussed, and suggestions for possible applications are given.
\end{abstract}

\section{Introduction}

Positioning materials and equipment accurately at a construction site is a fundamental process throughout the industry. The development of positioning systems such as the Global Positioning System (GPS) provides a new opportunity to bring robotic applications into the construction arena. Integrating robotic systems with existing positioning systems and construction machinery will be space-saving and cost-effective.

The key machinery used for lifting, transporting material and equipment, and positioning at the construction site is the crane. At present, a wide variety of cranes (such as crawler cranes, tower cranes, mobile cranes, and gantry cranes) perform a multitude of tasks. This makes the crane an outstanding tool from which to suspend workcells of all kinds. A precision crane with robotic integration could pick and place things symmetrically and asymmetrically. Examples of tasks completed by such a crane might be seen in several areas of the construction process to include bricklaying, painting, and inspection. Any unsafe or heavy-load highly precise operation is a good application for such a system.

However, common cranes have limitations for use with modern robotic systems. The currently flexible base provided by a crane is not suitable to hold a workcell securely since it does not provide the required stiffness. Cable adjustments would have to be made to reduce swinging and increase the accuracy in spatial positioning of tools where high precision is essential. Another necessary feature for positioning accuracy is electronic control of the suspension system. These adjustments and features are needed against perturbations such as moments, horizontal forces as windload, eccentric load, and the swinging motion caused by the attached workcell itself.

The Robotic Work Platform designed by the Construction Automation \& Robotics Lab (CARL) at North Carolina State University (NCSU) is an approach to kinematically constraining the suspension system and to develop a simple multipurpose high precision crane-suspended tool. 
The Robotic Work Platform is based on the Stewart-Gough Platform [Stewart 1965] and the RoboCrane technology [Bostelman et al. 1996] developed by the National Institute of Standards and Technology (NIST).

The Stewart Platform is a parallel link mechanism which is quite unique as it has six degrees of freedom and is kinematically constrained. It is capable of being positioned in cartesian space and oriented through roll, pitch, and yaw. Since the crane serves as a deployment system and the platform provides the fine positioning, its workspace can be located anywhere within the reach of a crane, even inside buildings. A major advantage is its ability to be designed for high lift-to-weight ratio, stable configurations, flexibility, and precise maneuverability.

\section{Design Concept}

The application of the Robotic Work Platform introduced here is an inverted Stewart Platform. It was designed using two parallel equilateral triangles referred to as lower and upper platform (Fig. 1). The lower platform is in rectangular order to provide space for power supplies, control hardware, and working teams. This allows the whole system to be self-contained. The mounting points of the pulleys are the vertices of an equilateral triangle with side length of $1829 \mathrm{~mm}$.

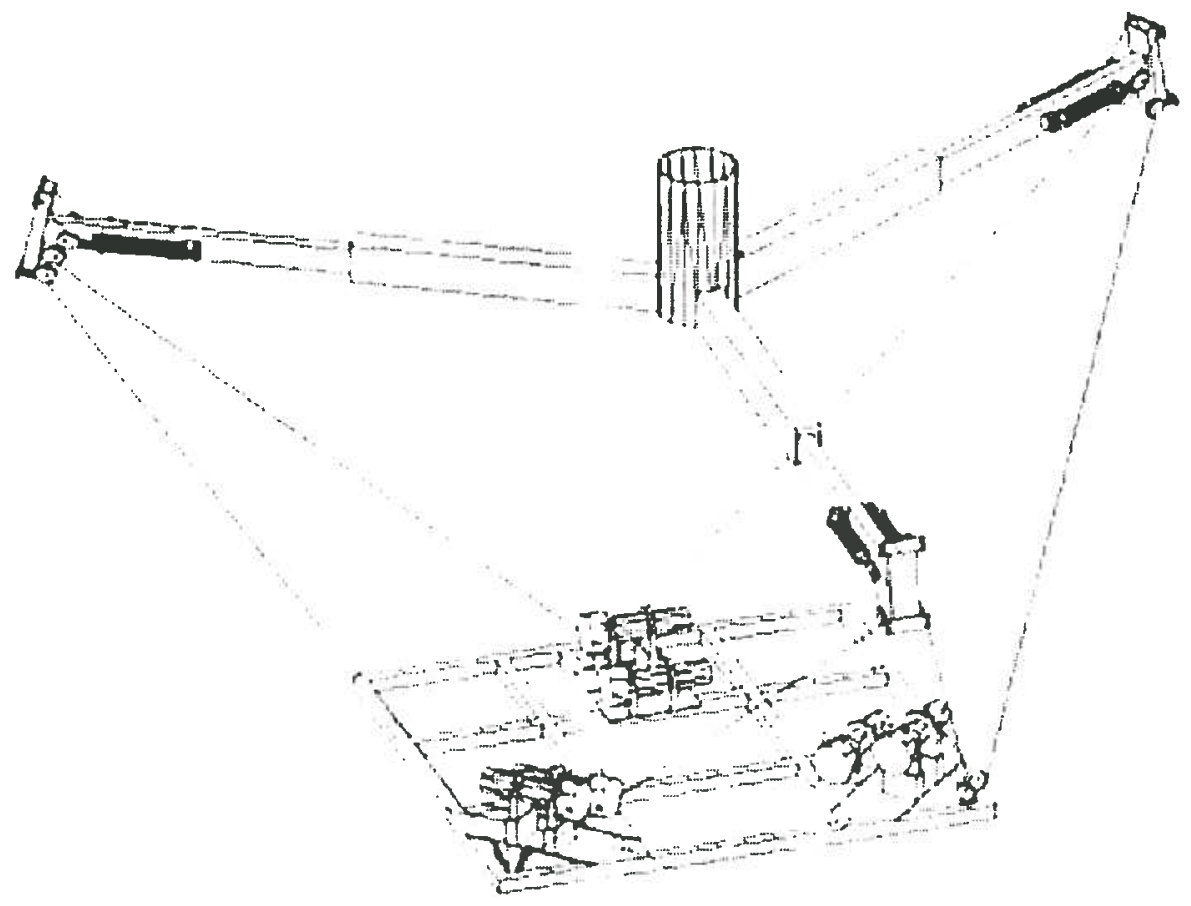

Figure 1: Design of the Cable Based Robotic Work Platform

The upper platform's structural frame consists of extendable booms (telescopic system) which makes its size variable. Different behaviors for various perturbations - such as moments, eccentric loads, and horizontal forces - make it necessary to adapt the platform to different demands to its stiffness properties. The telescopic system makes it possible to experimentally determine optimal dimensions of the upper platform in different tasks. The side length of the upper triangle can vary in a range from $3084 \mathrm{~mm}$ to 3962 $\mathrm{mm}$. 
The six degrees of freedom will be actuated via steel cables and six electric winches. For each cable a tension sensor is mounted to the upper platform to measure the tension in the cables (Figure 2). The tension in the cable extends the spring system and the springs' elongation can be measured with a potentiometer. Measuring the tension in the cables is required to have permanent information about the kinematic state of the mechanism. From this information the direction and amount of moment of inertia applied to the mechanism can be determined.

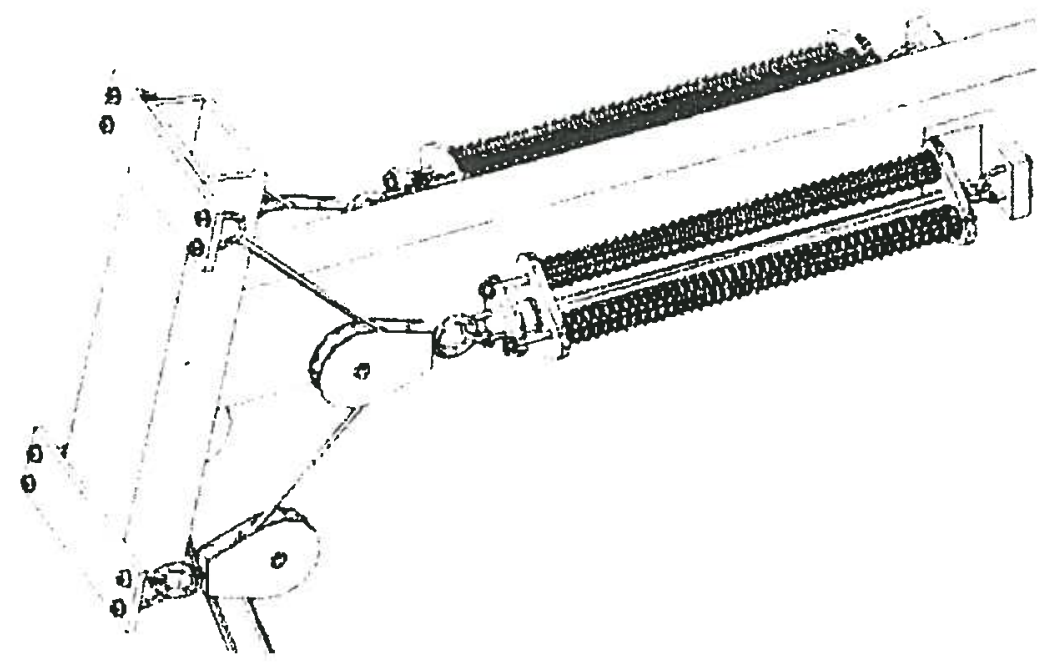

Figure 2: Cable Anchor Points at Upper Platform with Integrated Tension Sensors

The travel distance of each cable is measured by travel sensors attached to the lower platform to determine the amount of cable released or retracted. Each travel sensor constrains one cable in a way that the rotational information can be determined from a rotating pulley shaft using an absolute digital encoder (Figure 3). The measured travel distance is used to calculate the position and orientation of the lower platform in space.

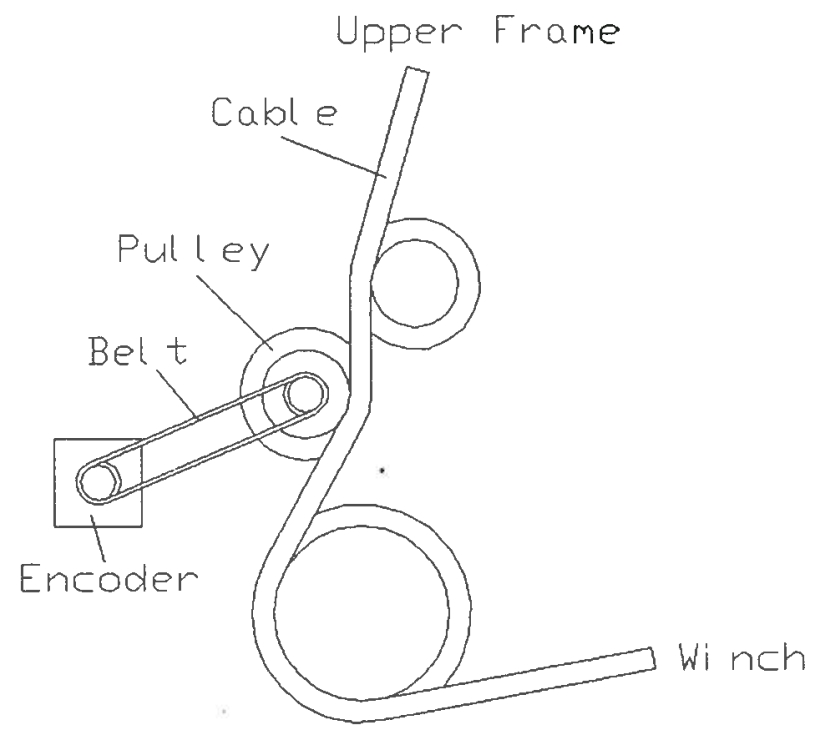

Figure 3: Travel Sensor 
For future field tests an automatic locking mechanism will be developed to make the Robotic Work Platform easily mountable to construction cranes. It is envisioned that the crane hook will be able to pick up the Robotic Work Platform and attach it to the crane by lifting it into the automatic locks. The locking mechanism will be standardized making it possible to easily interchange the Robotic Work Platform between overhead, tower, or mobile cranes.

The platform will also be self-contained with the power supplies, control boards, and winches directly mounted to the lower platform. It will be remotely controllable via an RF link.

\section{Control Concept}

\subsection{Hardware}

The control mechanisms consist of three parts: the measuring of the tension in the cables, the measuring of the travel of the cables, and the control of the winches.

All control algorithms run on a PC which is equipped with data acquisition hardware.

For the determination of the tension in the cables a spring system with potentiometers is being used as shown in Figure 2. The potentiometers are connected to an analog data acquisition board. The amount of potential at the input lines makes it possible to compute the elongation of the springs and from this the tension in each cable by using the following equation.

$$
\begin{aligned}
& F=\frac{k^{*} \Delta l}{a} \text { with } \\
& a=2 \sin \left(\arctan \frac{l_{y}}{l}+\arccos \frac{r}{\sqrt{l_{y}{ }^{2}+l^{2}}}\right)
\end{aligned}
$$

$l=\Delta l_{\max }-\Delta l$

$\mathrm{k}$ : $\quad$ spring constant $(8.755 \mathrm{~N} / \mathrm{mm})$

$\Delta \mathrm{l}$ : elongation of the springs

$l_{y}$ : design dependent, vertical distance between upper cable anchor point and center of upper swivel eye pulley $(155.6 \mathrm{~mm})$

r: design dependent; radius of the upper swivel eye pulley $(25.4 \mathrm{~mm})$

$\Delta \mathrm{l}_{\text {max }}$ : maximal elongation of springs $(101.6 \mathrm{~mm})$

To determine the amount of cable released or retracted by the winches, absolute digital encoders are used. These encoders are connected to a digital data acquisition board. The encoders provide 128 distinct positions per revolution which are transmitted via an 8 bit Graycode [Bourns 1998]. The encoder maps the output for the 128 (7 bit) positions to 8 bit (256 values). This avoids similarities in the bit patterns of neighboring positions. This Graycode has to be transformed into real position data by using a transformation table.

For the low level control of the winches a relay board connected to the parallel interface of the PC is used. 


\subsection{Software}

The software is written in $\mathrm{C}++$ and assembler language using Borland and Microsoft $\mathrm{C}++$ compilers. The software has three abstraction layers.

For the data acquisition hardware a class library was developed. The class library provides a well defined interface for user applications. In this part all the programming of the ports is done. This part of the software is the only part with direct hardware access and is called the hardware abstraction layer.

The application layer was implemented on top of the hardware abstraction layer. This part has no direct access to the hardware and provides basic functionality for user applications. It provides the transformation of the encoder's Graycode into real positions, calculation of the tension in the cables, and basic control functions for the winches. This layer is hardware independent.

The third layer is the user interface itself. In this layer the high level control algorithms will be implemented. It will provide a wide range of possibilities to control the Robotic Work Platform both manually and autonomously. It will involve a graphical interface under Windows $95^{\mathrm{TM}}$ or Windows NT $4.0^{\mathrm{TM}}$ for enhanced task planning and system tracking capabilities. It is planned to develop a path generator which can calculate control sequences to move the lower platform to any required location and orientation (given plane in space) within its workspace and even along interpolated paths to fulfill special tasks like welding. This layer also checks for cable slack which can occur if the platform crosses certain boundaries of its workspace. This checking is done both by evaluating the data retrieved from the tension sensors and by geometric analysis of the platform's current state.

Since the control algorithms have to run under real-time conditions the software is designed to be speed efficient. To provide a high input update cycle rate for the calculations of the trigonometric functions precalculated tables are used.

The manual control of the system will be done with a $3 \mathrm{D}$ joystick or equivalent input device (e.g. Spaceball ${ }^{\mathrm{TM}}$ ).

\subsection{Spatial Integration}

By polling the output of the encoders it will be possible to determine the exact position and orientation of the lower platform relative to coordinate system of the Robotic Work Platform. The update cycle of this system is constrained by the processor and bus speed of the PC only and lies approximately in a range of $20 \mathrm{~Hz}$.

As a secondary position measuring system a laser based spatial positioning system, ODYSSEY ${ }^{\mathrm{TM}}$ by SPSi, Inc., will be used. This system works like the GPS but provides higher accuracy. It will be used to monitor the position of the whole Robotic Work Platform with respect to a global coordinate system. The ODYSSEY ${ }^{\mathrm{TM}}$ consists of two laser transmitters in the environment and several laser receivers which can be mounted to the lower and the upper platform. Connected to a PC running AutoCAD, the 3D position and orientation of the platform can be monitored and controlled in real-time. A similar system has previously been used and tested for robotic excavation [Huang 1997]. The ODYSSEY $^{T M}$ system allows the data to be updated at a rate of $5 \mathrm{~Hz}$. 
This laser system supports the travel sensor system since with the travel sensors only positions relative to the Robotic Work Platform's coordinate system can be determined. The laser system will be used to determine the position of the Robotic Work Platform in a global coordinate system, since the laser transmitters are fixed in the environment. Additionally, the second system introduces fault tolerance to the Robotic Work Platform since it implements a secondary layer of feedback if at least two receivers are mounted to the lower platform. Each system can be used to verify the other's data.

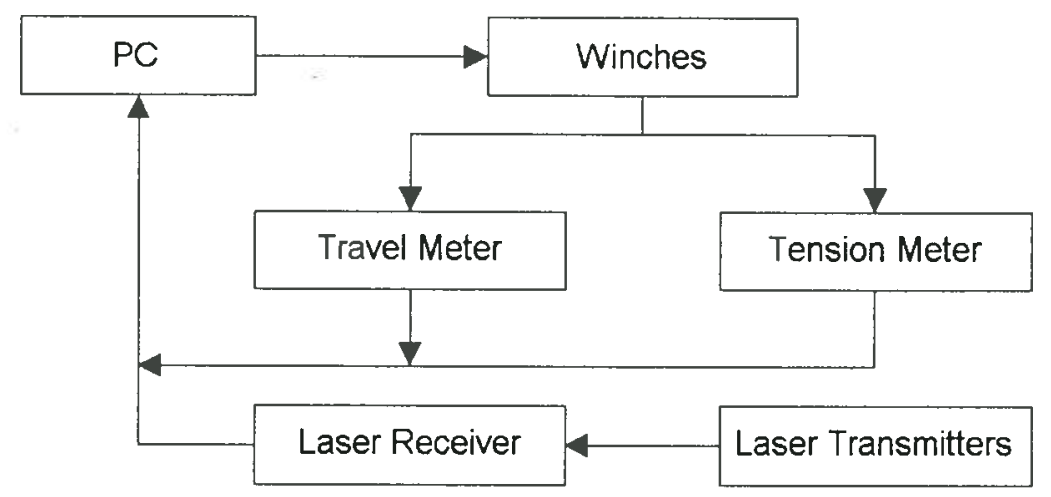

Figure 4: Control Flow Chart

\section{Applications in Construction}

The system is going to be used as part of a planned robotic testbed. This testbed is involved in CARL's research on Embodied Intelligence. The Robotic Work Platform will provide a base for various suspended robotic workcells. The first planned test setup using a masonry robot as the workcell is shown in Figure 5.

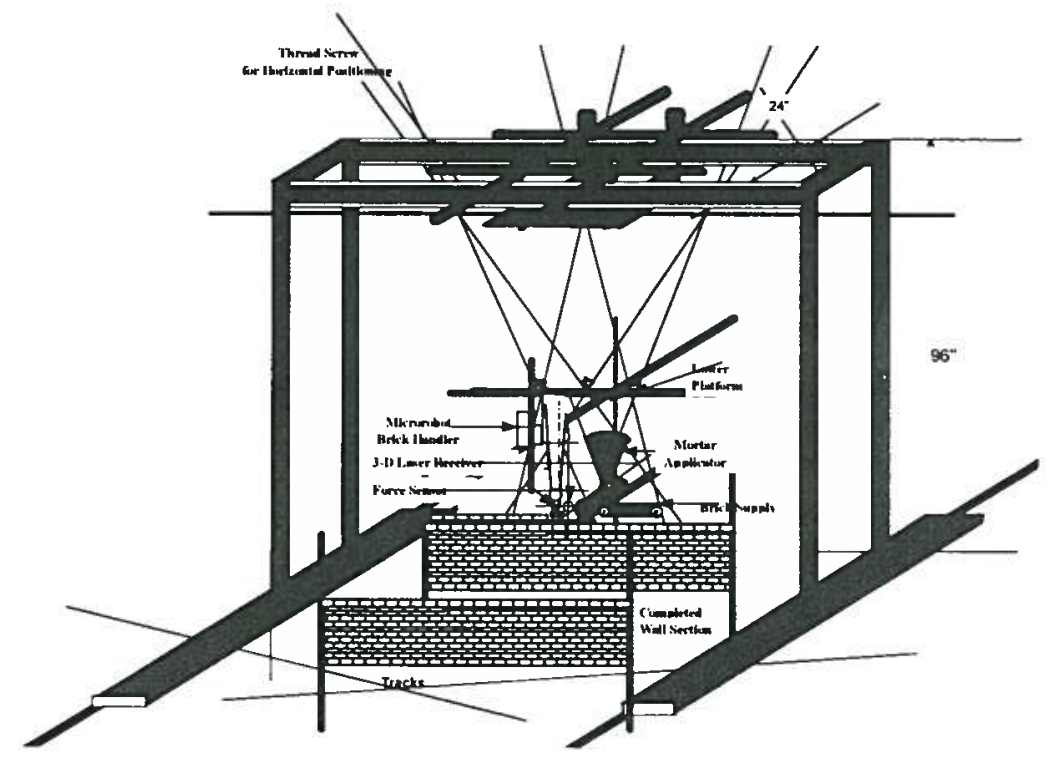

Figure 5: Conceptual Drawing of Robotic Masonry Testbed

The gantry crane will be used to position the suspended system - consisting of the Robotic Work Platform and a robotic masonry workcell - into its workspace while the Robotic Work Platform provides fine positioning and orientation of the workcell. 
The positioning table will be used for laboratory testing and is only one possible means of mounting the Robotic Work Platform and robotic workcell. The system is developed to be mounted on a crane for use at a construction site. By attaching this system to a crane, a mobile automated factory is created. This factory can be moved from site to site and built/disassembled as easily as driving an available crane to the work area. By attaching the Robotic Work Platform/workcell combination, the system is allowed to perform its tasks.

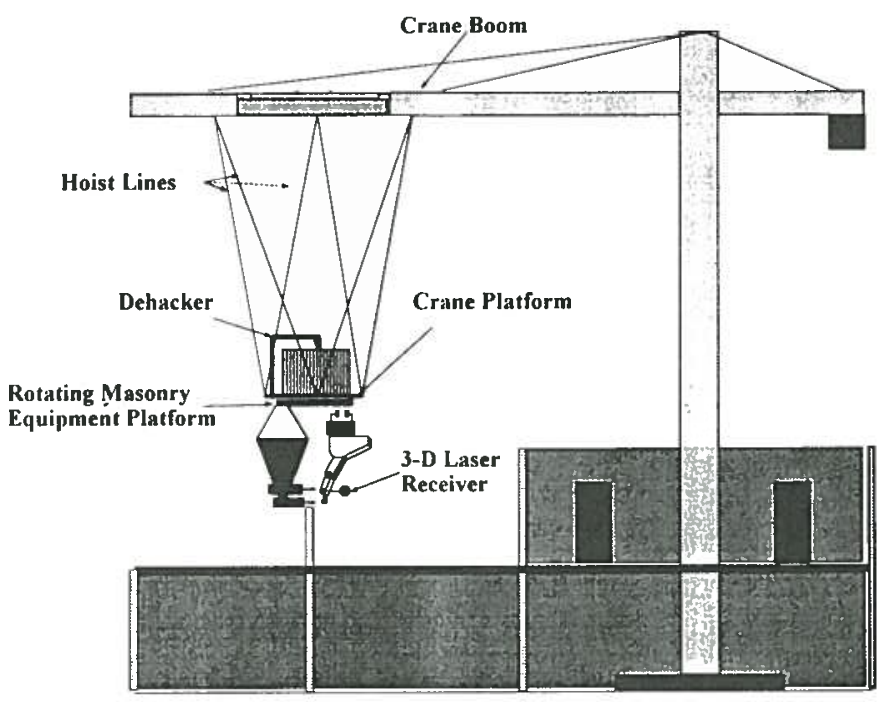

Figure 6: Robotic Work Platform mounted to a Tower Crane for On-Site Use

Since a construction site is a highly unstructured and constantly changing environment, the system must be intelligent and readily adaptable to the changes. This type of scenario leads to the need for embodied intelligence. Figures 6 and 7 show conceptual drawings of the Robotic Work Platform and robotic masonry workcell attached to the hook of a tower and crawler crane.

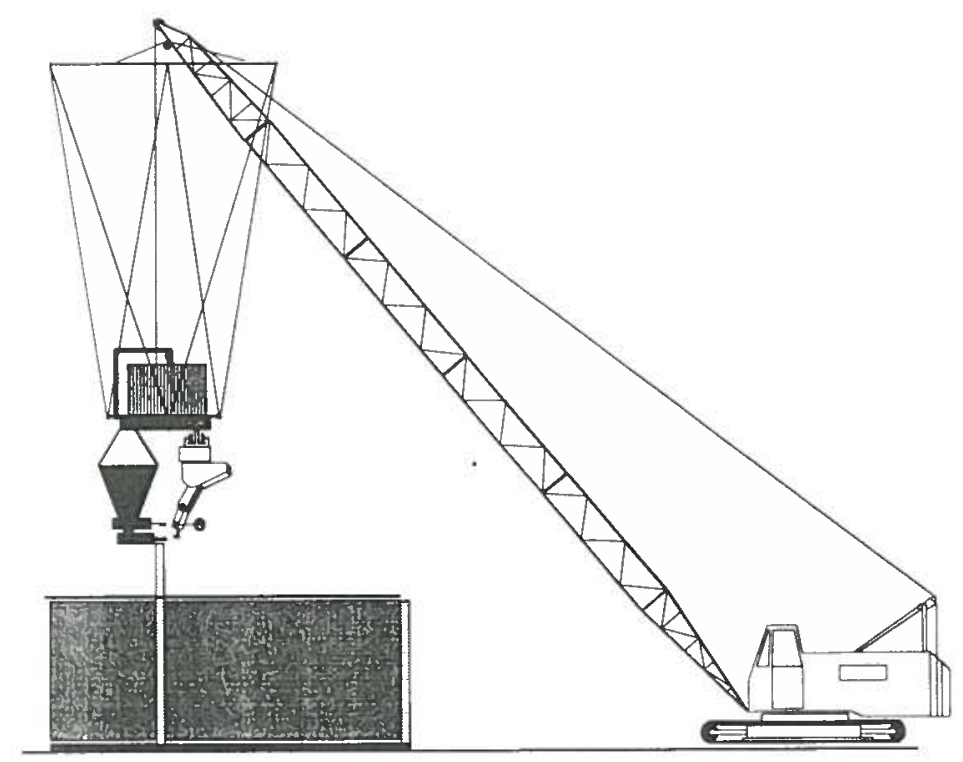

Figure 7: Robotic Work Platform mounted to a Crawler Crane for On-Site Use 
Since the testbed is a combination of three individual systems: a positioning table, a Robotic Work Platform, and a workcell, it will be necessary to make intelligence available to all three. For this reason, when a disturbance occurs, it will be necessary for the testbed to determine which of the three systems needs to compensate for the disturbance. In order to simplify this decision making, each system will be assigned a distinct function. The system can be considered as layered, where each layer leads into a higher precision of positioning of tools.

- The gantry crane will be used for positioning the Robotic Work Platform and the workcell attached to it within the workspace

- The Robotic Work Platform will be used for fine positioning of the workcell

- The robotic masonry workcell will be used for specific task applications, in this case, placement of bricks and application of mortar

Changes and disturbances which would be found on a construction site will be simulated for proper evaluation (e.g. wind gusts, dust, something hitting the testbed). The amount of time required to correct and the degree of accuracy will be measured. The testbed will compensate for any path deviation, position deviation, or any type of error. This ability is highly critical if an automated machine is to be used in a highly unstructured environment as a construction site. Additionally, safety of the system must be addressed. It is possible to have a human walk within the workspace, so the system must be capable of adapting to this situation. Since the testbed will have extensive information about the environment, its ability to adapt to this disturbance will also be tested.

\section{Conclusion}

The introduced inverted Stewart Platform application is not only capable of holding and positioning robotic applications. Its nature makes it an outstanding intelligent suspension and positioning tool for applications, other than on a construction site. The Robotic Work Platform will become an extension to any kind of crane to make it a high performance multipurpose tool by mounting a specialized workcell to the platform.

Since the crane hook remains independent after the Robotic Work Platform is locked, the Robotic Work Platform is capable of becoming a working base for teams to solve lots of tasks which now require additional equipment. Such tasks include welding of vertical pipes, vertical drilling, or maintenance of oil production sites.

It is even conceivable to use the system as an autonomous maintenance tool in contaminated areas such as nuclear facilities and waste areas.

\section{References}

Stewart, D. (1965). " A platform with six degrees of freedom" Proceedings of the Institution of Mechanical Engineers 1965-66, Vol.180 Pt.1 No.15 pp.371-378

Bostelman, R., Albus, J., Dagalakis, N., and Jacoff, A. (1996). "RoboCrane Project: An Advanced Concept for Large Scale Manufacturing" Proceedings of the Association for Unmanned Vehicles Systems International (AUVSI) Conference, Orlando, FL, July $15-$ 19,1996

Huang, X., Bernold, L. (1997). "CAD-Integrated Excavation and Pipe Laying" Journal of Construction Engineering and Management, September 1997

Bourns Inc., Datasheet of the ACE-128 Absolute Digital Encoder, 1998 